\title{
Prevalencia del síndrome metabólico en hipertensos estadio I
}

\author{
Daniela Urina-Jassir*, Miguel Urina-Triana, José Balaguera-Mendoza, \\ Luis Montenegro-Rolong, Manuel Urina-Jassir y Manuel Urina-Triana
}

Grupo de Investigación COL0019919 Centro de investigaciones Cardiodiagnóstico SA, Fundación del Caribe para la Investigación Biomédica, Universidad Simón Bolívar, Barranquilla, Colombia

Recibido el 15 de septiembre de 2015; aceptado el 28 de febrero de 2016

Disponible en Internet el 11 de mayo de 2016

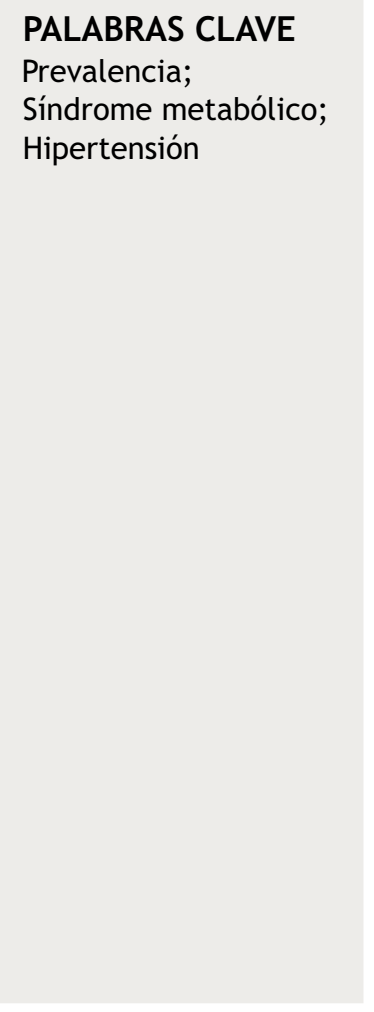

\begin{abstract}
Resumen
Introducción y Objetivo: En sujetos con hipertensión arterial sistémica recién diagnosticada es importante verificar la existencia de otros factores de riesgo para enfermedad cardiovascular para poder establecer estrategias para su óptimo tratamiento. Conocer cual es la prevalencia del síndrome metabólico en sujetos con hipertensión arterial sistémica estadio 1.

Métodos: Estudio prospectivo, observacional, transversal, realizado durante un año en Bogotá, Medellín, Cali y Barranquilla en sujetos de ambos géneros, $\geq 18$ años $\mathrm{y} \leq 60$ años con hipertensión arterial sistémica estadio 1 recién diagnosticada y sin tratamiento farmacológico. A todos los sujetos se les verificó la existencia de síndrome metabólico de acuerdo con el reciente consenso de la Federación Internacional de Diabetes, del Instituto Nacional del Corazón, Pulmón y Sangre, de la Asociación Americana del Corazón, de la Federación Mundial del Corazón, de la Sociedad Internacional de Aterosclerosis y de la Asociación Internacional para el Estudio de la Obesidad.

Resultados: La prevalencia promedio de síndrome metabólico en la población total de hipertensos estudiada fue de $16.84 \%$. En Cali fue de $28.8 \%$, en Medellín fue $18.9 \%$, en Barranquilla fue $12.5 \%$ y en Bogotá fue 11.4\%. Medellín tuvo la más alta prevalencia en hombres (14.7\%) y Cali la mas alta en mujeres (15.2\%).

Conclusiones: En los sujetos con hipertensión arterial sistémica de reciente diagnóstico se recomienda realizar una búsqueda sistemática del síndrome metabólico. En las ciudades estudiadas de Colombia existió gran variabilidad en la prevalencia del síndrome metabólico encontrada. Se requieren estudios para conocer en otras ciudades de Colombia cual es la prevalencia real del síndrome metabólico en sujetos con hipertensión arterial sistémica en el pais e implementar estrategias educativas para minimizar esta.

๑ 2016 Sociedad Colombiana de Cardiología y Cirugía Cardiovascular. Publicado por Elsevier España, S.L.U. Este es un artículo Open Access bajo la licencia CC BY-NC-ND (http:// creativecommons.org/licenses/by-nc-nd/4.0/).
\end{abstract}

\footnotetext{
* Autor para correspondencia.

Correo electrónico: danielaurina@fundacionbios.org (D. Urina-Jassir).
} 


\section{KEYWORDS}

Prevalence;

Metabolic syndrome;

Hypertension

\section{Prevalence of metabolic syndrome in stage । hypertensive patients}

\begin{abstract}
Introduction and motivation: It is important for recently diagnosed systemic hypertension (HPB) individuals to verify the existence of other cardiovascular risk factors, in order to establish optimal treatment strategies. Knowing the prevalence of metabolic syndrome (MS) in patients with stage $1 \mathrm{HBP}$.

Methods: Prospective, observational, cross-sectional study conducted over one year in Bogotá, Medellin, Cali and Barranquilla in individuals of both genders, $\geq 18$ and $\leq 60$ years old with recently diagnosed stage $1 \mathrm{HBP}$ and without drug therapy. Presence of MS was verified for all subjects according to recent consensus of the International Diabetes Federation, the National Heart, Lung and Blood Institute, the American Heart Association, the World Heart Federation, the International Atherosclerosis Society and the International Association for the Study of Obesity.

Results: Average prevalence of MS in the total population of hypertensive patients was of $16.84 \%$. In Cali it was of $28.8 \%$, in Medellin of $18.9 \%$, in Barranquilla of $12.5 \%$ and in Bogotá of $11.4 \%$. Medellin showed the highest prevalence in men $(14.7 \%)$ and Cali in women $(15.2 \%)$. Conclusions: It is recommended to conduct a systematic search for MS in patients who have recently been diagnosed with HBP. Prevalence among MS in the studies Colombian cities varied greatly. More studies are required to find out about the real prevalence of MS in patients with HBP in other Colombian cities and to implement educational studies to minimise its impact.

(C) 2016 Sociedad Colombiana de Cardiología y Cirugía Cardiovascular. Published by Elsevier España, S.L.U. This is an open access article under the CC BY-NC-ND license (http:// creativecommons.org/licenses/by-nc-nd/4.0/).
\end{abstract}

\section{Introducción}

Las enfermedades cardiovasculares son la principal causa de la morbilidad y la mortalidad en Colombia, con una tasa estimada de mortalidad de 130.2 por 100.000 habitantes $^{1}$. En casi el $90 \%$ de los sujetos con diagnóstico de hipertensión arterial sistémica (HTA) no es posible establecer una etiología ${ }^{2}$. La literatura mundial ha sugerido la importancia y la relación existente entre el incremento del índice de masa corporal (IMC) y el aumento del perímetro abdominal (PA), como un factor fisiopatológico importante en la génesis de la HTA. Desde el año 1988, Reaven y Hoffman ${ }^{3}$, caracterizaron los componentes de un síndrome que con el pasar del tiempo ha demostrado estar asociado con el incremento de riesgo cardiovascular y que estaría compuesto por: resistencia a la insulina, intolerancia a la glucosa, hipersinsulinemia, triglicéridos elevados, reducción del colesterol HDL e HTA. La prevalencia del síndrome metabólico (SM) y de la diabetes mellitus, se incrementa con la edad ${ }^{4}$. La presencia de SM es un predictor importante en el desarrollo de la diabetes mellitus ${ }^{5}$ y en sujetos con hipertensión arterial es algo que siempre debería investigarse. El objetivo principal del presente estudio fue caracterizar una población urbana ambulatoria en cuatro grandes ciudades de Colombia (población urbana), de sujetos adultos entre 18 a 60 años con diagnóstico de HTA estadio । de la clasificación del "Seventh Report of the Joint National Committee on Prevention, Detection, Evaluation, and Treatment of High Blood Pressure" (JNC 7) ${ }^{6}$, sin diabetes mellitus ni insuficiencia renal y conocer en ellos la prevalencia del síndrome metabólico, su distribución por ciudad, por género, por edad y por raza.
Como objetivos secundarios fueron planteados: 1 . Conocer el perímetro abdominal (PABD). 2. Conocer el indice de masa corporal (IMC) promedio de la población estudiada. 3. Conocer la prevalencia de tabaquismo y de consumo de alcohol. 4. Conocer los antecedentes hereditarios de la HTA. 5. Conocer la actividad física diaria y su asociación con la presencia del SM. 6. Conocer la prevalencia del SM en las mujeres con pre y postmenopausia.

\section{Materiales y métodos}

Estudio prospectivo, observacional, transversal, realizado entre diciembre de 2013 a diciembre de 2014, en 4 ciudades de Colombia: Bogotá, Medellín, Cali y Barranquilla.

Para la selección de los médicos encargados de evaluar a la población estudiada se realizó una convocatoria abierta en la página de Médicos Generales de Colombia. https://www.medicosgeneralescolombianos.com. En esta convocatoria se invitó a los médicos generales de: Bogotá, Cali, Medellín y Barranquilla a participar en un proyecto de investigación en hipertensión arterial. A la convocatoria respondieron 374 médicos (115 de Bogotá, 110 de Barranquilla, 85 de Cali y 74 de Medellín). Se seleccionaron por cada ciudad a los primeros 50 médicos que cumplieron con los siguientes requisitos: 1 . Deseo de participar voluntariamente y por escrito en el estudio. 2. Tener su práctica clínica y estar radicados en la ciudad elegida. 3. Estar debidamente certificados ante el ente territorial de salud respectivo (debieron aportar el acto resolutorio de habilitación de su consultorio). 4. Experiencia clínica profesional (definida, con más de 5 años de haber egresado de su facultad de medicina). 5. Que en su práctica clínica de consulta 
externa atendiera pacientes con el diagnóstico de hipertensión arterial sistémica. 6. Presentar los certificados de calibración vigente de la balanza y del tensiómetro de su consultorio.

Después de la selección se realizaron reuniones en las cuatro ciudades con los médicos seleccionados, en las cuales se les capacitó: 1. En buenas prácticas clínicas, con énfasis en el informe de consentimiento. 2. En el protocolo de investigación por parte de la Fundación del Caribe para la Investigación Biomédica. 3. En la plataforma virtual a través de la cual debían diligenciar el cuadernillo de recogida de datos y sobre los datos que debían ser consignados. 4. En el proceso y metodología de la auditoría de los datos y la cronología de visitas por una firma externa independiente (Trial \& Train Ltda.). 5. Sobre los tiempos de fecha de inicio y fecha de terminación del estudio. 6. En la estandarización en la toma de la presión sanguínea según el video publicado en el año 2009, por el New England Journal of Medicine (http://www. nejm.org/doi/full/10.1056/NEJMvcm0800157). 7. En la estandarización de la medición del perímetro abdominal. Se incluyeron sujetos de ambos géneros, con edad $\geq 18$ años $y \leq$ 60 años con diagnóstico de HTA recién diagnosticada estadio I sin tratamiento farmacológico que no tuvieran diagnóstico previo de diabetes mellitus tipo 1 o 2, (a todos los sujetos se les realizó glucometría en ayunas, excepto a los que traían un resultado de glicemia en ayunas realizado en los 3 meses previos y que esta fuera $<126 \mathrm{mg} / \mathrm{dl}$ ) y sin enfermedad renal aguda o crónica. En las mujeres se tuvo en cuenta la presencia de menopausia definida como el cese permanente de las menstruaciones durante al menos 12 meses consecutivos.

El diagnóstico de la HTA estadio I se hizo conforme a las recomendaciones del $7 \mathrm{JNC}^{6}$, cuando el sujeto tuviera en 3 ocasiones diferentes realizadas en la consulta externa, medidas de las cifras de presión sanguínea así: cifras de tensión arterial sistólica (TAS) $\geq 140 \mathrm{~mm} \mathrm{Hg} \mathrm{y} \leq 159 \mathrm{mmHg}$ o tensión arterial diastólica (TAD) $\geq 90 \mathrm{~mm} \mathrm{Hg}$ y $\leq 99$ o ambas $\geq 140 / 90 \mathrm{mmHg}$. Se usó para el diagnóstico del SM la presencia de al menos tres de los cinco criterios propuestos por el reciente consenso de la Federación Internacional de Diabetes, del Instituto Nacional del Corazón, Pulmón y Sangre, de la Asociación Americana del Corazón, de la Federación Mundial del Corazón, de la Sociedad Internacional de Aterosclerosis y de la Asociación Internacional para el Estudio de la Obesidad ${ }^{7}$ y que son: a) Circunferencia de cintura elevada, de acuerdo a la población y al país especifico que se estudie; b) Triglicéridos elevados de más de $150 \mathrm{mg} / \mathrm{dl}$, o recibir tratamiento para hipertrigliceridemia; c) HDL menor de $40 \mathrm{mg} / \mathrm{dl}$ en hombres y menor de $50 \mathrm{mg} / \mathrm{dl}$ en mujeres (o recibir tratamiento para un HDL bajo como ácido nicotínico); d) Tensión arterial alta normal o en rango de hipertensión (TAS $130 \mathrm{mmHg}$ y/o TAD $85 \mathrm{mmHg}$ o recibir tratamiento antihipertensivo); y e) Glucosa en ayunas mayor de $100 \mathrm{mg} / \mathrm{dl}$ o recibir tratamiento para la elevación de la glucosa plasmática. Muchos autores consideran que la obesidad central abdominal es el principal factor y que siempre debe estar presente para hacer el diagnóstico del SM, por esta razón en este estudio este criterio siempre tuvo que estar presente para hacer el diagnóstico. Para Colombia y en general en Latinoamérica el perímetro abdominal se considera normal cuando es menor de $90 \mathrm{cms}$ para los hombres y menor de $80 \mathrm{cms}$ para las mujeres y así lo usamos en este estudio ${ }^{8}$, además, a todos los sujetos se les realizó el índice de masa corporal (IMC).

Se revisaron los antecedentes de dislipidemia (hipercolesterolemia, hipertrigliceridemia o ambas) según la clasificación del ATP III ${ }^{9}$, si el sujeto tenía realizado un perfil lipídico (colesterol total, HDL y triglicéridos), en los tres meses previos este fue utilizado y si no lo tenía este le fue solicitado. Se evaluó el antecedente familiar de hipertensión arterial de los padres. También se interrogó el factor de riesgo de adicción al tabaco (calculando el consumo de cigarrillos al día) y el antecedente del consumo de alcohol (calculando los gramos de alcohol consumidos utilizando la siguiente fórmula: volumen expresado en centímetros cúbicos (cc) x graduación (\%) x 0.8/100); para este estudio se definió consumo ocasional como el consumo $\leq$ a una ocasión al mes y consumo en gramos de alcohol $<27$ gramos/día. Se indagó al sujeto sobre la frecuencia de su actividad física así: si hacía ejercicio de 1 - 3 días o de 4 - 6 días por semana o toda la semana. El análisis estadístico se realizó utilizando el paquete SPSS versión 20 (SPSS Inc, Chicago, Illiois USA).

\section{Resultados}

La población estudiada fue de 1.021 sujetos con reciente diagnóstico de hipertensión arterial sistémica estadio I (Clasificación según el 7 JNC) (hombres 541 (53\%) y mujeres 480 (47\%)) con edad promedio de 47.6 \pm 9.1 (Rango: 18-60 años) y con una distribución por raza así: mestizo el 87,8\% (897), blancos el $12 \%(123)$ y asiático $0.2 \%$ (2), (ver tabla 1$)$.

Con relación a sus antecedentes personales encontramos: la hipercolesterolemia en el $26 \%(n=273)$, la hipertrigliceridemia en el $21.6 \%(n=221)$ y la dislipidemia mixta en el $15 \%$ $(n=154)$, el $72.57 \%(n=741)$ tenía antecedentes familiares de hipertensión arterial sistémica en el padre, la madre o en ambos.

Con relación a la cifras de tensión arterial, la cifra promedio de tensión arterial sistólica fue de $146,2 \pm 6.4 \mathrm{mmHg}$ y la de tensión arterial diastólica fue de 92,6 $\pm 4.1 \mathrm{mmHg}$. La frecuencia cardiaca promedio medida fue de $79 \pm 7.6$ latidos por minuto.

En la tabla 2 vemos el análisis de la suma de criterios encontrados para el diagnóstico del síndrome metabólico (SM). Hay que tener en cuenta que para el diagnóstico del síndrome metabólico siempre debe estar presente el criterio de perímetro abdominal. En la población que estudiamos, además, de este criterio presente en todos los sujetos diagnosticados con el SM también tenían presente el criterio de hipertensión arterial sistémica, por tanto, lo que se revisó fue la presencia de estos dos criterios y el de los otros utilizados para el diagnóstico del SM (triglicéridos, glicemia, HDL), entendiendo que para el diagnóstico del síndrome metabólico se requería de la presencia de al menos 3 criterios. Los 3 criterios que se presentaron más frecuentemente fueron: el perímetro abdominal (Pabd), la hipertensión arterial (HTA) y la hipertrigliceridemia (TG > $150 \mathrm{mgdl}$ ) en el $16.8 \%$ de la población, seguidos del Pabd, la HTA y la glicemia (glicemia $\geq 100 \leq 125 \mathrm{mgdl}$ ) en el $11,25 \%$ y del Pabd, la HTA y el HDL ( $\mathrm{HDL}<50$ mujeres $\mathrm{y}<40$ mgdl en hombres) en el 9,92\%. Los 4 criterios de Pabd, HTA, Glicemia y HDL estuvieron presentes en el $8.5 \%$ de la población y los 5 criterios estuvieron 
Tabla 1 Características de la población estudiada

\begin{tabular}{ll}
\hline Edad promedio en años & $47,6 \pm 9,1$ \\
Total (n) & $1021(100 \%)$ \\
Género & \\
$\quad$ Hombres & \\
$\quad$ Mujeres & $541(53 \%)$ \\
$\quad$ Edad fértil & $480(47 \%)$ \\
$\quad$ Menopausia & $205(42 \%)$ \\
Raza & $275(58 \%)$ \\
$\quad$ Mestiza & \\
$\quad$ Blanca & \\
$\quad$ Asiática & $87,8 \%$ \\
Hipercolesterolemia & $12 \%$ \\
Hipertrigliceridemia & $0,2 \%$ \\
Dislipidemia mixta & $273(26 \%)$ \\
Tabaquismo & $221(21,6 \%)$ \\
Consumo promedio cigarrillos/día & $154(15 \%)$ \\
Exfumadores & $139(14 \%)$ \\
Consumo de alcohol 1 vez a la semana & $18,5 \pm 12,5$ \\
Consumo promedio en gramos & $224(22 \%)$ \\
Consumo ocasional de alcohol & $148(14,5 \%)$ \\
Antecedente de HTA en los padres & $27,5 \pm 17,3$ \\
Presión sistólica sanguínea promedio & $54(5,3 \%)$ \\
Presión diastólica sanguínea promedio & $741(72,6 \%)$ \\
Frecuencia cardiaca promedio & $146,2 \pm 6,4$ \\
IMC promedio & $92,6 \pm 4,1$ \\
Perímetro abdominal promedio & $79 \pm 7.6$ \\
Actividad física (Ejercicio) Días/semana & $27,98 \pm 4,2$ \\
$\quad$ 1-3 días/semana & $92,84 \pm 14,4$ \\
$4-6$ & \\
7 & $350(34,28 \%)$ \\
$\quad$ Sedentarios & $96(9,4 \%)$ \\
\hline HT: hipertension arterial sistemica; & $33(3,23 \%)$ \\
& $542(53,09 \%)$ \\
\hline
\end{tabular}

HTA: hipertensión arterial sistémica; IMC = índice de masa corporal. presentes en el $7.05 \%$ de la población. Se encontró el SM en 84 hombres $(15,52 \%)$ y en 88 mujeres $(18,3 \%)$.

En la tabla 3 vemos la prevalencia del SM distribuida por ciudad así: Barranquilla 389 sujetos con prevalencia de $4,7 \%$ y ajustada por ciudad de $12,5 \%$, Bogotá 287 sujetos con prevalencia de 3,3\% y ajustada por ciudad de $11,5 \%$, Cali 250 sujetos con prevalencia de $7,05 \%$ y ajustada por ciudad de $28,8 \%$ y Medellín 95 sujetos con prevalencia de $1,76 \%$ y ajustada por ciudad de $18,9 \%$. Con relación al género masculino Medellín obtuvo la más alta prevalencia ajustada $(27,45 \%)$ y Cali con un $32,2 \%$ la obtuvo para el género femenino.

En la tabla 4 vemos la prevalencia del SM por el género y la edad. Observamos que en el intervalo de 40 a 49 años y el de 50 a 60 años, se encontró la mayor prevalencia ajustada por el grupo etario del síndrome metabólico $(13,96 \%$ y $22,31 \%$, respectivamente), $(p<0,001)$.

En la tabla 5 vemos la prevalencia del SM por la raza. La prevalencia ajustada por la raza mestiza el $17,2 \%$ y por la raza Blanca el 13,8\%, ( $<<0,001)$.

En la tabla 6 observamos la distribución del perímetro abdominal según el género, el 70,06\% de los hombres y el 79.37\% de la mujeres tuvieron un valor del perímetro abdominal por encima del valor normal. El perímetro abdominal promedio de la población estudiada fue $92.8 \mathrm{cms}$, el promedio del género masculino fue $95.4 \mathrm{cms}$ y el del género femenino $89.6 \mathrm{cms}$. El índice de masa corporal (IMC) en promedio de la población estudiada fue de 27,98 \pm 14.4 .

Se encontró la presencia del tabaquismo en el $14 \%$ $(n=139)$ de la población estudiada con una media de $18.5 \pm 12.5$ cigarrillos al día y un $22 \%$ de exfumadores. $\mathrm{El}$ $14,5 \%$ de la población consumía alcohol una vez a la semana con una media de $27,5( \pm 17,3)$ gramos de alcohol ingeridos y el $5.3 \%$ lo consumía ocasionalmente (una o menos veces al mes).

Las mujeres estuvieron distribuidas de acuerdo con su carácter reproductivo así: edad fértil 205 (42\%) y menopausia 275 (58\%), la prevalencia del SM en mujeres fue mayor en las menopaúsicas $(21,09 \%)$ que con la edad fértil $(14,73 \%)$, $(\mathrm{p}<0,001)$, (ver tabla 7).

Tabla 2 Suma de criterios para diagnóstico de síndrome metabólico (SM)

\begin{tabular}{|c|c|c|c|c|c|}
\hline \multirow[t]{2}{*}{ CRITERIOS } & \multirow{2}{*}{$\begin{array}{l}\text { Número de criterios } \\
\mathrm{n}\end{array}$} & \multicolumn{2}{|c|}{ Hombres $(n=541)$} & \multicolumn{2}{|c|}{ Mujeres $(n=480)$} \\
\hline & & $\mathrm{n}$ & $\%$ & $\mathrm{n}$ & $\%$ \\
\hline \multicolumn{6}{|l|}{ No SM } \\
\hline $\mathrm{Pabd}^{*}+\mathrm{HTA}^{* *}$ & 2 & 369 & 70.1 & 381 & 79.4 \\
\hline \multicolumn{6}{|l|}{ SM } \\
\hline Pabd + HTA + TG & 3 & 84 & 15.52 & 88 & 18.3 \\
\hline Pabd + HTA + Glicemia & 3 & 50 & 9.2 & 64 & 13.3 \\
\hline $\mathrm{Pabd}+\mathrm{HTA}+\mathrm{HDL}^{* * * * *}$ & 3 & 49 & 9.05 & 52 & 10.8 \\
\hline Pabd + HTA + Glicemia + HDL & 4 & 42 & 7.24 & 47 & 9.79 \\
\hline Pabd + HTA + Glicemia + HDL + TG & 5 & 34 & 6.2 & 38 & 7.9 \\
\hline
\end{tabular}

SM: síndrome metabólico.

* Pabd: perímetro abdominal Hombres $>90 \mathrm{cms}$, Mujeres $>80 \mathrm{cms}$.

${ }^{* *}$ HTA: hipertensión arterial.

${ }^{* * *}$ TG: Triglicéridos $>150$ mgdl, Glicemia: Glicemia $\geq 100<126$.

**** HDL: Mujeres $<50$ y Hombres $<40$ mgdl. 
Tabla 3 Prevalencia del síndrome metabólico (SM) por ciudades

\begin{tabular}{|c|c|c|c|c|c|}
\hline Ciudades & Hombres & Mujeres & Total & $\begin{array}{l}\text { Prevalencia de acuerdo con el } \\
\text { numero total de sujetos de la } \\
\text { muestra }(n=1021)\end{array}$ & $\begin{array}{l}\text { Prevalencia } \\
\text { ajustado por } \\
\text { ciudad }\end{array}$ \\
\hline \multicolumn{6}{|l|}{ Cali } \\
\hline Sin SM & 98 & 80 & 178 & & \\
\hline Con SM & 34 & 38 & 72 & $7,05 \%$ & $28,8 \%$ \\
\hline $\mathrm{n}$ & 132 & 118 & 250 & & \\
\hline \multicolumn{6}{|l|}{ Medellín } \\
\hline Sin SM & 37 & 40 & 77 & & \\
\hline Con SM & 14 & 4 & 18 & $1,76 \%$ & $18,9 \%$ \\
\hline $\mathrm{n}$ & 51 & 44 & 95 & & \\
\hline \multicolumn{6}{|c|}{ Barranquilla } \\
\hline Sin SM & 191 & 149 & 340 & & \\
\hline Con SM & 15 & 34 & 49 & $4,8 \%$ & $12,5 \%$ \\
\hline $\mathrm{n}$ & 206 & 183 & 389 & & \\
\hline \multicolumn{6}{|l|}{ Bogotá } \\
\hline Sin SM & 131 & 123 & 254 & & \\
\hline Con SM & 21 & 12 & 33 & $3,23 \%$ & $11,5 \%$ \\
\hline $\mathrm{n}$ & 152 & 135 & 287 & & \\
\hline Total & & & & $16,84 \%$ & \\
\hline
\end{tabular}

Tabla 4 Prevalencia del síndrome metabólico (SM) por género y por edad

\begin{tabular}{|c|c|c|c|c|c|}
\hline Edad en años & Hombres & Mujeres & Total & $\begin{array}{l}\text { Prevalencia de acuerdo al } \\
\text { tamaño de muestra }(n=1021)\end{array}$ & $\begin{array}{l}\text { Prevalencia } \\
\text { ajustada a grupo } \\
\text { etario }\end{array}$ \\
\hline \multicolumn{6}{|l|}{$18-29$} \\
\hline Sin SM & 23 & 17 & 40 & & \\
\hline Con SM & 1 & 2 & 3 & $0,29 \%$ & $6,25 \%$ \\
\hline $\mathrm{n}$ & 28 & 20 & 48 & & \\
\hline \multicolumn{6}{|l|}{$30-39$} \\
\hline Sin SM & 77 & 62 & 139 & & \\
\hline Con SM & 9 & 4 & 13 & $1,28 \%$ & $8,33 \%$ \\
\hline $\mathrm{n}$ & 91 & 65 & 156 & & \\
\hline \multicolumn{6}{|l|}{$40-49$} \\
\hline Sin SM & 165 & 108 & 273 & & \\
\hline Con SM & 20 & 24 & 44 & $4,30 \%$ & $13,96 \%$ \\
\hline $\mathrm{n}$ & 183 & 132 & 315 & & \\
\hline \multicolumn{6}{|l|}{$50-60$} \\
\hline Sin SM & 192 & 205 & 397 & & \\
\hline Con SM & 54 & 58 & 112 & $10,97 \%$ & $22,31 \%$ \\
\hline $\mathrm{n}$ & 239 & 263 & 502 & & \\
\hline Total & & & & $16,84 \%$ & \\
\hline
\end{tabular}

En la tabla 8 vemos la prevalencia del SM con relación a la actividad física, el 34,38\% de los hombres hacia ejercicio de 1-3 veces a la semana, el 9,43\% de $4-6$ veces y el $3,14 \%$ toda la semana, el resto tuvo sedentarismo de $53,05 \%$. Con relación a las mujeres, el $32,3 \%$ hacia ejercicio de $1-3$ veces a la semana, el $11,45 \%$ de $4-6$ semanas y el 3,13\% 7 días a la semana y el $53,12 \%$ eran sedentarias. Cuando revisamos la prevalencia del SM ajustada con relación a la actividad física, esta fue mayor en los sujetos sedentarios $28,04 \%$ vs.
$4,17 \%$ en los sujetos que realizaban algún tipo de actividad física, $(p<0,001)$.

\section{Discusión}

En América Latina la enfermedad cardiovascular es una de las principales causas de la mortalidad y causa alrededor de 800.000 muertes por año ${ }^{10,11}$. La tasa de muerte por la 
Tabla 5 Prevalencia del síndrome metabólico (SM) por raza

\begin{tabular}{|c|c|c|c|c|c|}
\hline Raza & Hombres & Mujeres & Total & $\begin{array}{l}\text { Prevalencia de } \\
\text { acuerdo con } \\
\text { tamaño de } \\
\text { muestra }(n=1021)\end{array}$ & $\begin{array}{l}\text { Prevalencia } \\
\text { ajustada de } \\
\text { acuerdo con la } \\
\text { raza }\end{array}$ \\
\hline \multicolumn{6}{|l|}{ Mestizo } \\
\hline Sin SM & 399 & 342 & 741 & & \\
\hline Con SM & 76 & 79 & 155 & $15,18 \%$ & $17,2 \%$ \\
\hline $\mathrm{n}$ & 475 & 421 & 896 & & \\
\hline \multicolumn{6}{|l|}{ Blanco } \\
\hline Sin SM & 57 & 49 & 106 & & \\
\hline Con SM & 8 & 9 & 17 & $1,66 \%$ & $13,8 \%$ \\
\hline $\mathrm{n}$ & 65 & 58 & 123 & & \\
\hline \multicolumn{6}{|l|}{ Asiático } \\
\hline Sin SM & 1 & 1 & 2 & & \\
\hline Con SM & 0 & 0 & 0 & 0 & 0 \\
\hline $\mathrm{n}$ & 1 & 1 & 2 & & \\
\hline Total & & & & $16,84 \%$ & \\
\hline
\end{tabular}

Tabla 6 Perímetro abdominal según género

\begin{tabular}{lclll}
\hline & \multicolumn{3}{c}{ Perímetro abdominal } \\
\cline { 2 - 5 } & Normal $^{*}$ & $\%$ & Anormal $^{* *}$ & $\%$ \\
\hline Hombres & 162 & 29,94 & 379 & 70,06 \\
Mujeres & 99 & 20,62 & 381 & 79,37 \\
Total & 261 & $\mathbf{2 5 , 5 7}$ & $\mathbf{7 6 0}$ & $\mathbf{7 4 , 4 3}$ \\
\hline
\end{tabular}

*Normal $<90 \mathrm{~cm}$ en hombres $\mathrm{y}<80 \mathrm{~cm}$ mujeres.

** Anormal $\geq 90 \mathrm{~cm}$ en hombres $\mathrm{y} \geq 80 \mathrm{~cm}$ en mujeres.

Tabla 7 Prevalencia del síndrome metabólico (SM) mujeres pre y postmenopausia

\begin{tabular}{lllll}
\hline Mujeres & $\mathrm{n}$ & Con SM & $\begin{array}{l}\text { Prevalencia de acuerdo } \\
\text { con tamaño de muestra }\end{array}$ & $\begin{array}{l}\text { Prevalencia de acuerdo con } \\
\text { pre y posmenopausia }\end{array}$ \\
\hline Edad Fértil (premenopausia) & 205 & 30 & $\mathbf{2 , 9 3 \%}$ & $\mathbf{1 4 , 7 3 \%}$ \\
Postmenopausia & 275 & 58 & $5,68 \%$ & $21,09 \%$ \\
Total & 480 & 88 & $\mathbf{8 , 6 1 \%}$ & $\mathbf{1 8 , 3 3 \%}$ \\
\hline
\end{tabular}

enfermedad cardiaca isquémica y el accidente cerebrovascular viene en aumento y se espera que se triplique en el año $2020^{10}$, por eso se ha dicho que América Latina se encuentra en la fase inicial de una epidemia de la enfermedad cardiovascular $^{12}$.

La expectativa de vida de la población de América Latina viene en aumento, pero también lo hace la prevalencia de la hipertensión arterial sistémica (HTA), de la obesidad, de la diabetes mellitus y del síndrome metabólico ${ }^{12-15}$, producto de los cambios en los estilos de vida como lo son: el mayor sedentarismo y el consumo de dietas hipercalóricas con gran contenido de grasa. La prevalencia de estos factores de riesgo (HTA, obesidad y diabetes) en América Latina, se ha convertido en similar a la observada en los países más desarrollados $^{12,16,17}$. Se espera que el total de muertes estimadas en 23 países en desarrollo va a aumentar y casi la mitad de estas muertes se producirán en personas menores de 70 años en comparación con solo el $27 \%$ en los países de altos ingresos ${ }^{18}$. Esta cifra se elevará al $53 \%$ en el año 2030, con una cuota global de la carga de morbilidad ajustada por discapacidad/años de vida de casi el 60\%. A largo plazo se produciría un gran ahorro económico, si se logrará una reducción del $2 \%$ anual adicional en las tasas de mortalidad por enfermedades crónicas, según lo recomendado por la OMS y se ahorrarían casi en un $10 \%$ la pérdida esperada en los ingresos en estos países ${ }^{19}$.

La HTA es un riesgo cardiovascular en América Latina y su prevalencia con relación a otros factores de riesgo cardiovasculares varía ampliamente en toda la región, probablemente, debido a una serie de situaciones, como son las diferencias en la incidencia del tabaquismo y la obesidad y la variación étnica. Por otra parte, los estudios 
Tabla 8 Prevalencia de síndrome metabólico (SM) y actividad física

\begin{tabular}{|c|c|c|c|c|c|}
\hline $\begin{array}{l}\text { Actividad Física } \\
\text { (días/semana) }\end{array}$ & Hombres & Mujeres & Total & $\begin{array}{l}\text { Prevalencia de acuerdo con } \\
\text { tamaño de la muestra }\end{array}$ & $\begin{array}{l}\text { Prevalencia ajustada a la } \\
\text { actividad física }\end{array}$ \\
\hline \multicolumn{6}{|l|}{$1-3$} \\
\hline Sin SM & 180 & 146 & 326 & & \\
\hline Con SM & 6 & 9 & 15 & $1,47 \%$ & $4,39 \%$ \\
\hline $\mathrm{n}$ & 186 & 155 & 341 & & \\
\hline \multicolumn{6}{|l|}{$4-6$} \\
\hline Sin SM & 49 & 54 & 103 & & \\
\hline Con SM & 2 & 1 & 3 & $0,29 \%$ & $2,83 \%$ \\
\hline $\mathrm{n}$ & 51 & 55 & 106 & & \\
\hline \multicolumn{6}{|l|}{7} \\
\hline $\operatorname{Sin} S M$ & 16 & 14 & 30 & & \\
\hline Con SM & 1 & 1 & 2 & $0,19 \%$ & $6,25 \%$ \\
\hline $\mathrm{n}$ & 17 & 15 & 32 & & \\
\hline \multicolumn{6}{|c|}{ Actividad física (todos) } \\
\hline Sin SM & 245 & 214 & 459 & & \\
\hline Con SM & 9 & 11 & 20 & $1,95 \%$ & $4,17 \%$ \\
\hline $\mathrm{n}$ & 254 & 225 & 479 & & \\
\hline \multicolumn{6}{|l|}{ Sedentarismo } \\
\hline Sin SM & 212 & 178 & 390 & & \\
\hline Con SM & 75 & 77 & 152 & $14,89 \%$ & $28,04 \%$ \\
\hline $\mathrm{n}$ & 287 & 255 & 542 & & \\
\hline Total & & & & $16,84 \%$ & \\
\hline
\end{tabular}

epidemiológicos en América Latina han sido difíciles de comparar por inconsistencias metodológicas, como las definiciones de factores de riesgo, las variaciones en la edad, las técnicas de muestreo pobres y los métodos de evaluación ${ }^{20}$. Un estudio realizado en sujetos entre 25 a 64 años de edad en siete ciudades de América Latina informó una prevalencia de la HTA que varió desde un mínimo de $9 \%$ en Quito (Ecuador), hasta un 24 a 29\% en Santiago (Chile), Barquisimeto (Venezuela) y Buenos Aires (Argentina) ${ }^{21}$. En este último país, en la ciudad de Córdoba, otro estudio informó una prevalencia de la HTA de $30 \%$ en sujetos entre 15 a 85 años de edad ${ }^{22}$. El diagnóstico y manejo de la HTA en América Latina en general, sigue las directrices del Comité Nacional Conjunto ${ }^{6}$, de la OMS y de la Sociedad Internacional de Hipertensión (ISH) ${ }^{23}$ y de la Sociedad Europea de Cardiología (ESC) ${ }^{24}$. Aproximadamente el $80 \%$ de la carga de enfermedades atribuibles a la hipertensión arterial ocurre en los países de bajos y medianos ingresos (incluida América Latina), con tasas de mortalidad atribuible de 1,5 a 2 veces mayor en las regiones de bajos o medianos ingresos en comparación con la de altos ingresos. Una mayor proporción de esta carga de la enfermedad se presenta en personas más jóvenes. Aproximadamente la mitad de esta carga es en las personas con una PAS $<145$ $\mathrm{mmHg}^{17}$. Muchos países, como Argentina, Brasil, Colombia, Ecuador, México, Perú y Venezuela, han desarrollado directrices propias y se ha publicado al menos en un consenso latinoamericano en hipertensión y un consenso latinoamericano en diabetes e hipertensión ${ }^{25-27}$, pero a pesar de ello las tasas de control de la presión sanguínea son inaceptablemente bajas en Latinoamérica, con tasas tan bajas como el $13 \%$ y el $15 \%$ en Argentina y Cuba, respectivamente ${ }^{22,28}$. Por otra parte, la inversión financiera inadecuada en la asistencia sanitaria representa una barrera adicional a la gestión exitosa para el control de la hipertensión en Latinoamérica. Por ejemplo, mientras que el $11,6 \%$ de la carga mundial de muerte y discapacidad por cualquier causa se atribuye a los países desarrollados, estos países representan más del $90 \%$ de los gastos de atención médica ${ }^{29}$. La hipertensión debe competir con otras enfermedades crónicas que tienen menos impacto en la morbilidad y la mortalidad y en los gastos de atención médica, y esto puede dar lugar a una distribución desproporcionada de los fondos en relación con los resultados de la salud ${ }^{30}$. Estas observaciones tristes, justifican una llamada a la acción para mejorar el control de la HTA y otros factores de riesgo cardiovasculares en toda Latinoamérica. La consecución de estos ambiciosos objetivos requerirá esfuerzos y colaboración de muchos grupos, entre ellos el gobierno, los responsables políticos, las organizaciones internacionales, los proveedores de salud, las escuelas y la sociedad ${ }^{31}$. No hay que olvidar que la enfermedad cardiovascular es un continuo evolutivo que comienza con la aparición de factores de riesgo y si estos no son controlados evolucionan a la muerte prematura por la enfermedad cardiovascular o situaciones incapacitantes como la falla cardiaca ${ }^{32}$. En forma reciente los resultados del estudio PURE, donde participaron cuatro países de Latinoamérica, mostró una prevalencia de la HTA para Colombia del $37.5 \%{ }^{33}$. La prevalencia del síndrome metabólico en el continente americano puede sufrir grandes variaciones, poblaciones como la de los Estados Unidos han aumentado la prevalencia de la obesidad abdominal particularmente en las mujeres ${ }^{34}$. Una revisión sistemática del síndrome metabólico en América Latina en aparentemente sujetos sanos, entre 18 a 65 años de edad, mostró una prevalencia del 
mismo del 24,9\% (rango: 18,8-43,3) con una diferencia entre las mujeres $(25,3 \%)$ y los hombres $(23,2 \%)$ y con mayor prevalencia en sujetos mayores de 50 años de edad. En esta revisión el componente que más acompañó al criterio del perímetro abdominal elevado presente en el $45,8 \%$ fue el del HDL (62,9\%), el estudio llama la atención sobre el pobre o ningún dato que se tiene de algunos países ${ }^{35}$. En el año 2013 una revisión realizada en el Brasil mostró una prevalencia del síndrome metabólico del 29,6\% (rango: 14,9$65,3 \%)$, con una alta prevalencia $(65,3 \%)$ en la población indígena y más baja en la población rural $(14,9 \%)$. Los componentes más frecuentes que acompañaron a la obesidad abdominal en este estudio fueron el HDL con el 59,3\% y la hipertensión arterial con el $52,5 \%^{36}$. En Colombia tenemos varios estudios, pero muy segmentados de poblaciones particulares con una muy diferente prevalencia del síndrome metabólico ${ }^{37-45}$, en uno de ellos donde fueron utilizadas varias definiciones para el síndrome metabólico (SM) la prevalencia osciló entre $12,3 \%$ a $34,9 \%$, variación que fue bastante llamativa ${ }^{37}$.

Por otro lado, además, de conocer la prevalencia de la HTA y de su asociación con el SM, está el interrogante de ¿qué hacer con los sujetos menores de 60 años de edad portadores de hipertensión estadio । y que tengan síndrome metabólico? Los grados de recomendación para el tratamiento de sujetos hipertensos menores de 60 años de edad fueron discutidos con la mejor evidencia disponible en el 8 Joint National Committee $(\mathrm{JNC})^{46}$. En vista de que el riesgo cardiovascular es alto en los pacientes hipertensos con síndrome metabólico, es necesario hacer un riguroso control de la presión arterial y a no ser que existan indicaciones específicas, en los pacientes con el síndrome metabólico se debe evitar el uso de betabloqueadores, ya que son bien conocidos sus efectos adversos en el aumento de peso, en la incidencia de nuevos casos de diabetes, en la resistencia a la insulina y en el perfil lipídico. Los diuréticos tiazídicos presentan efectos diabetogénicos y otras acciones dismetabólicas, especialmente a dosis altas, por lo que no deben utilizarse en pacientes con el síndrome metabólico. Por tanto, los fármacos recomendados como primera opción en sujetos hipertensos con el síndrome metabólico son los inhibidores de la enzima convertidora de angiotensina (IECA) o los antagonistas de los receptores de angiotensina (ARA ॥), los cuales han demostrado reducir la incidencia de nuevos casos de diabetes y tener también efectos favorables en el daño del órgano blanco ${ }^{47}$. Si no se controla la presión sanguínea con monoterapia, a estos se les deberá asociar un bloqueador de los canales de calcio ${ }^{48}$, combinación que produce una menor incidencia de nuevos casos de diabetes que la combinación con betabloqueadores o diuréticos tiazídicos.

En este estudio la población de hipertensos estudiada mostró una prevalencia del SM del $16,84 \%$, en estos el sedentarismo, la edad (mayores de 40) y en la mujeres la condición de postmenopaúsica son los factores que condicionan su mayor prevalencia.

El análisis de la prevalencia por ciudades en el presente estudio fue muy difícil y no tenemos una explicación muy clara para la existencia de la mayor prevalencia ajustada en algunas más que en las otras de las ciudades estudiadas. Lamentablemente, una explicación que podría ser la raza, dado que el síndrome metabólico es mayor en las poblaciones afrodescendientes, no nos permite saber si en Cali que fue donde encontramos mayor prevalencia, existe dentro de la raza mestiza, un mayor mestizaje de la población de afrodescendientes con mayor prevalencia de la HTA y del SM. Este hallazgo deberá ser planteado como hipótesis para la realización de un nuevo estudio especifico para responder ese interrogante. Por otro lado, somos conscientes que nuestro estudio es observacional y tiene limitaciones, el no haber utilizado una muestra aleatoria de sujetos con la HTA hace que la forma de escogencia de la muestra plantee un sesgo importante para responder la diferencia entre las prevalencias aunque para contrarrestar esta posibilidad, los pacientes se inscribieran con independencia de la presencia de factores de riesgo para el SM. Estudios previos han demostrado que la asociación entre los factores de riesgo metabólico y el pobre control de la presión sanguínea en este tipo de sujetos, por lo que identificar en sujetos con la HTA criterios del SM, es muy útil para establecer una mejor estrategia de tratamiento ${ }^{49}$.

\section{Conclusiones}

Es muy importante que en todos los sujetos diagnosticados con la hipertensión arterial sistémica realizarles la búsqueda en forma sistemática y constante del diagnóstico del síndrome metabólico. En las cuatro ciudades de Colombia estudiadas se requieren de la implementación de estrategias educativas para minimizar la prevalencia del síndrome metabólico ${ }^{50}$ y aunque existen estudios sobre la prevalencia del síndrome metabólico en varias ciudades de Colombia ${ }^{51}$, aún necesitamos conocer más sobre la prevalencia del síndrome metabólico en sujetos con hipertensión arterial. Además, en nuestro país, se requiere de la implementación de más estudios sobre el origen del incremento del SM y sobre ¿cuál es el papel del ambiente y de la epigénetica? Una reciente publicación ${ }^{52}$, atribuye el aumento de las tasas de la hipertensión, del síndrome metabólico y de la diabetes mellitus tipo 2 que se observa en América Latina, al resultado de la discrepancia entre el entorno nutricional durante la vida fetal y el medio ambiente para los adultos; asimismo, este Consenso Latinoamericano propone estimular a la academia para desarrollar la investigación dirigida para establecer los mecanismos epigenéticos que explican la relación entre la malnutrición materna, la restricción del crecimiento temprano y la aparición posterior de la obesidad abdominal y la enfermedad cardiovascular en Latinoamérica, por tanto, aun queda un gran camino por recorrer para el entendimiento de esta enfermedad.

\section{Responsabilidades éticas}

Protección de personas y animales. Los autores declaran que para esta investigación no se han realizado experimentos en seres humanos ni en animales.

Confidencialidad de los datos. Los autores declaran que en este artículo no aparecen datos de pacientes.

Derecho a la privacidad y consentimiento informado. Los autores declaran que en este artículo no aparecen datos de pacientes. 


\section{Conflictos de intereses}

Los autores declaran no tener conflictos de interés en el presente trabajo.

\section{Reconocimientos y agradecimientos}

A todos los médicos generales que en las cuatro ciudades de Colombia nos apoyaron con el reclutamiento y la evaluación de los sujetos del estudio.

\section{Bibliografía}

1. Encuesta Nacional de Salud. Ministerio de la Protección Social. Colombia 2007 y https://www.minsalud.gov.co/Documentos\% 20y\%20Publicaciones/ENCUESTA\%20NACI ONAL.pdf

2. Carretero O, Oparil S. Essential hypertension. Part I: definition and etiology. Circulation. 2000;01(3):329-35.

3. Reaven G, Hoffman B. A role for insulin in the etiology and course of hypertension? Lancet. 1987;2:435-7.

4. Grant R, Meigs J. Should the insulin resistance syndrome be treated in the elderly? Drugs Aging. 2004;21:141-51.

5. Lorenzo C, Williams K, Hunt K, Haffner SM. The National Cholesterol Education Program- Adult Treatment Panel III, International Diabetes Federation, and World Health Organization definitions of the metabolic syndrome as predictors of incident cardiovascular disease and diabetes. Diabetes Care. 2007;30:8-13.

6. Chobanian A, Bakris G, Black H, Cushman WC, Green LA, Izzo Jl, et al., and the National High Blood Pressure Education Program Coordinating Committee. Seventh Report of the Joint National Committee on Prevention, Detection, Evaluation, and Treatment of High Blood Pressure. Hypertension. 2003;42:1206-52.

7. Alberti K, Eckel R, Grundy S, Zimmet PZ, Cleeman JI, Donato KA, et al. Harmonizing the metabolic syndrome. A Joint Interim Statement of the International Diabetes Federation Task Force on Epidemiology and Prevention; National Heart, Lung, and Blood Institute; American Heart Association; World Heart Federation; International Atherosclerosis Society; and International Association for the Study of Obesity. Circulation. 2009;120:1640-5.

8. Aschner P, Buendía R, Brajkovich I, Gonzalez A, Figueredo R, Juarez XE, et al. Determination of the cut-off point for waist circumference that establishes the presence of abdominal obesity in Latin American men and women. Diabetes Res Clin Pract. 2011;93:243-7.

9. Third Report of the National Cholesterol Education Program (NCEP) Expert Panel on Detection, Evaluation, and Treatment of High Blood Cholesterol in Adults (Adult Treatment Panel III) Final Report Circulation. 2002;106:3143.

10. Yach D, Hawkes C, Gould C, Hofman KJ. The global burden of chronic diseases: overcoming impediments to prevention and control. JAMA. 2004;91(21):2616-22, 2.

11. Mozaffarian D, Benjamin E, Go A, Amett DK, Blaha MJ, Cushman M, de Ferranti S, et al. AHA Statistical Update: Heart Disease and Stroke Statistics-2015 Update: A Report From the American Heart Association. Circulation. 2015;31:e29-322.

12. Cubillos-Garzón LA, Casas JP, Morillo CA, Bautista L. Congestive heart failure in Latin America the next epidemic. Am Heart J. 2004;47(3):412-7.

13. Royer M, Castelo-Branco C, Blumel JE, Chedraui PA, Danckers L, Bencosme A, et al. The US National Cholesterol Education Programme Adult Treatment Panel III (NCEP ATP III): prevalence of the metabolic syndrome in postmenopausal Latin American women. Climacteric. 2007;10(2):164-70.
14. Bustos P, da Silva AA, Amigo H, Bettiol H, Barbieri MA. Metabolic syndrome in young adults from two socioeconomic Latin American settings. Nutr Metab Cardiovasc Dis. 2007;17(8):581-9.

15. James WP. The epidemiology of obesity: the size of the problem. J Intern Med. 2008;263(4):336-52.

16. Hernández-Hernández R, Armas-Padilla MC, Armas-Hernández MJ, Velasco M. Hypertension and cardiovascular health in Venezuela and Latin American countries. J Hum Hypertens. 2000;14 Suppl.1:S2-5.

17. Burt VL, Whelton P, Roccella EJ, Brown C, Cutler JA, Higgins M, et al. Prevalence of hypertension in the US adult population: results from the Third National Health and Nutrition Examination Survey, 1988-1991. Hypertension. 1995;25(3):305-13.

18. Lawes CM, Vander Hoorn S, Rodgers A. Global burden of blood pressure related disease, 2001. Lancet. 2008;371(9623):1513-8, 3.

19. Abegunde DO, Mathers CD, Adam T, Ortegon M, Strong K. The burden and costs of chronic diseases in low-income and middleincome countries. Lancet. 2007;370(9603):1929-38, 8.

20. Orduñez P, Silva LC, Rodríguez MP, Robles S. Prevalence estimates for hypertension in Latin America and the Caribbean: are they useful for surveillance? Rev Panam Salud Pública. 2001;10(4):226-31.

21. Schargrodsky H, Hernández-Hernández R, Champagne BM, Silva $H$, Vinueza R, Silva Aycaguer LC, et al. CARMELA: assessment of cardiovascular risk in seven Latin American cities. Am J Med. 2008;121(1):58-65.

22. Nigro D, Vergottini JC, Kuschnir E, Bendersky M, Campo I, De Roiter HG, et al. Epidemiología de la hipertensión arterial en la ciudad de Córdoba, Argentina. Rev Fed Arg Cardiol. 1999;28:1969-75.

23. Whitworth JA. World Health Organization (WHO)/International Society of Hypertension (ISH) statement on management of hypertension. J Hypertens. 2003;21(11):1983-92.

24. Mancia G, De Backer G, Dominiczak A, Cifkova R, Fagard R, Germano G, et al. ESH-ESC practice guidelines for the management of arterial hypertension: ESH-ESC Task Force on the Management of Arterial Hypertension. J Hypertens. 2007;25(9):1751-62.

25. Consenso de Hipertensión, Arterial., Consejo Argentino de Hipertensión, Arterial. Rev Arg, Cardiol. 2007;75 Suppl.3:1-43.

26. Ribeiro AB, Zanella MT, Kohlmann O Jr. Guidelines for treatment of hypertension in Latin America: the Brazilian experience. Clin Exp Hypertens. 1999;21(5-6):683-92.

27. Burlando G, Sánchez RA, Ramos FH, Mogensen CE, Zanchetti A. Latin American consensus on diabetes mellitus and hypertension. J Hypertens. 2004;22(12):2229-41.

28. Fuentes R, Ilmaniemi N, Laurikainen E, Tuomilehto J, Nissinen A. Hypertension in developing economies: a review of populationbased studies carried out from 1980 to 1998. J Hypertens. 2000;18(5):521-9.

29. Murray CJ, López AD. Global mortality, disability, and the contribution of risk factors: Global Burden of Disease Study. Lancet. 1997;349(9063):436-1442.

30. Arredondo A, Zúñiga A. Epidemiologic changes and economic burden of hypertension in Latin America: evidence from México. Am J Hypertens. 2006;19(6):553-9.

31. Rubinstein A, Alcocer L, Chagas A. High blood pressure in Latin America: a call to action. Ther Adv Cardiovasc Dis. 2009;3(4):259-85.

32. Alcocer L, Bendersky M, Acosta J, Urina-Triana M. Use of calcium cannel Blockers in Cardiovascular risk Reduction. Issues in Latin America. Am J Cardiovasc Drugs. 2010;10(3):143-54.

33. Chow Ck, Teo KK, Rangarajan S, Islam S, Gupta R, Avezum A, et al. PURE (Prospective Urban Rural Epidemiology) Study Investigators. Prevalence, awareness, treatment and control of hypertension in rural and urban communities en high, middle and low-income countries. JAMA. 2013;310:959-68. 
34. Beltrán-Sánchez H, Harhay MO, Harhay MM, McElligott S. Prevalence and Trends of Metabolic Syndrome in the Adult U.S. Population, 1999-2010. J Am Coll Cardiol. 2013;62(8):697-703.

35. Márquez F, Macedo G, Viramontes D, Fernandez Ballart JD, Salas-Salvado J, Vizmanos B. The prevalence of metabolic syndrome in Latin America: a systematic review. Public Health Nutrition. 2011;14:1702-13.

36. Carvalho F, Bressan J, Babio N, Salas-Salvado J. Prevalence of metabolic syndrome in Brazilian adults: a systematic review. BMC Public Health. 2013;13:1198.

37. Pinzón JB, Serrano N, Díaz A, Matilla G, Velasco HM, Martinez LX, et al. Impacto de la nuevas definiciones en la prevalencia del síndrome metabólico en una población adulta de Bucaramanga, Colombia. Biomédica. 2007;27:172-9.

38. Dávila E, Quintero M, Orrgeo M, Ford E, Walke H, Arenas M, Pratt $M$. Prevalence and risk factors for metabolic síndrome in Medellin and surrounding municipalities, Colombia 2008-2010. Preventive Medicine. 2013;56:30-4.

39. Ruiz AJ, Aschner PJ, Puerta MF, Alfonso-Cristancho R. Estudio IDEA (International Day for Evaluation of Abdominal Obesity): prevalencia de obesidad abdominal y factores de riesgo asociados en atención primaria en Colombia. Biomédica. 2012;32(4):610-6.

40. Navarro E, Vargas R. Riesgo coronario según ecuación de Framingham en adultos con síndrome metabólico de la ciudad de Soledad, Atlántico, 2010. Rev Colomb Cardiol. 2012;19(3):109-18.

41. Mari A, Marval Y, Suárez A, Arteaga E, Martinez E, Bastidas G. Síndrome metabólico en individuos de una comunidad rural. Acta Médica Colombiana. 2012;37(4):177-82.

42. Polo-Acosta P, Romero-Ucrós F, Saumeth-Bovea A, Urina-Triana M, Zuluaga-De Leon I, Alvis-Guzman N. Riesgo cardiovascular y síndrome metabólico en pacientes VIH positivos en el Caribe colombiano. Acta Médica Colombiana. 2013;38(4):222-7.
43. Paternina A, Alcalá G, Paillier J, Romero-Zarante A, AlvisGuzman N. Concordancia de tres definiciones de síndrome metabólico en pacientes hipertensos. Rev. Salud Pública. 2009;11(6):898-908.

44. Navarro E, Vargas R. Síndrome metabólico en el suroccidente de Barranquilla (Colombia). Salud Uninorte. 2008;24(1):40-52.

45. Mantilla M, Herazo J, Urina-Triana M. Factores de riesgo cardiovascular según género en el programa Muévete corazón de Barranquilla, 2011. Arch Med. 2014;14(1):21-8.

46. James PA, Oparil S, Carter BL, Cushman W, Dennison -Himmelfarb C, Handler J, et al. 2014 Evidence-Based Guideline for the Management of High Blood Pressure in Adults: Report From the Panel Members Appointed to the Eighth Joint National Committee (JNC 8). JAMA. 2014;311(5):507-20.

47. López-Jaramillo P. Tratamiento de la hipertensión arterial en el paciente con síndrome metabólico. Rev. Colomb. Cardiol. 2010;17:22-7.

48. Urina M, Vergara J. Bloqueadores de los canales de Calcio. Rev Colomb Cardiol. 2007;13(1):224-31

49. Cortez-Dias N, Martins S, Belo A, Fiuza M. On behalf of VALSIM Study Investigators. Association of metabolic risk factors with uncontrolled hypertension: comparison of the several definitions of metabolic syndrome. J Hypertens. 2013;31:1991-7.

50. López-Jaramillo P, Luengas C, Jaramillo N. Síndrome metabólico. En Guías de prevención primaria en riesgo cardiovascular. Rev Colomb Cardiol. 2009;16(3):75-82.

51. Manzur F, Alvear C, Alayon A. Caracterización fenotípica y metabólica del síndrome metabólico en Cartagena de Indias. Rev Colomb Cardiol. 2008;15:97-101.

52. López-Jaramillo P, Sánchez R, Díaz M, Cobos L, Bryce A, ParraCarrillo J, et al., on behalf of the Latin America Expert Group. Latin American consensus on hypertension in patients with diabetes type 2 and metabolic syndrome. J Hypertens. 2013;31(2):223-38. 